\title{
Dimensional crossover in half-filled and lightly doped $N$-leg Hubbard ladders
}

\author{
Urs Ledermann \\ Theoretische Physik, Eidgenössische Technische Hochschule, CH-8093 Zürich, Switzerland
}

(November 7, 2018)

\begin{abstract}
We study the dimensional crossover from 1D to 2D in half-filled and lightly doped, weakly interacting $N$-leg Hubbard ladders. In this case, the Hubbard ladders are equivalent to a $N$-band model. Using renormalization group techniques, we find, that the half-filled ladders exhibit (in the spin-sector) an odd-even effect only below a crossover energy $E_{c} \propto \exp [-\alpha \exp (\gamma N)](\alpha \ll 1$ and $\gamma \sim 1$ depend on the interaction strength and on the hopping matrix elements): Below $E_{c}$, the dominant interactions take place within band pairs $(j, N+1-j)$ [and within the band $(N+1) / 2$ for $N$ odd], such that even-leg ladders are an insulating spin-liquid, while odd-leg ladders have one gapless spinon-mode. In contrast, above the energy-scale $E_{c}$, all bands are interacting with each other and the system is a 2D-like (insulating) antiferromagnet; we obtain an analytical expression for the Hamiltonian which is similar to the 2D Heisenberg antiferromagnet. Bosonization techniques show, that in the charge-sector the Mott insulator is as well below and above $E_{c}$ of the same type as in $N / 2$ half-filled two-leg ladders. Doping away from half-filling, we find that the effect of an increasing doping $\delta$ is very similar to decreasing the number of legs $N$ : In both cases interactions between unpaired bands are suppressed and thereby the antiferromagnetic correlations reduced. The resulting band pairs form then insulating spin-liquids and when doped, there is a spin-gap, but phase coherence exists only within the band pairs. At higher doping levels $\delta_{c}=\delta_{c}(N)$, phase coherence between all band pairs sets in and the system becomes a 2D-like $d$-wave superconductor $\left[\delta_{c}(N) \rightarrow 0\right.$ for $\left.N \rightarrow \infty\right]$.

PACS numbers: 71.10.Pm, 74.20.Mn
\end{abstract}

\section{INTRODUCTION}

The physical properties of (quasi-)one-dimensional electron systems are often very different from their $2 \mathrm{D}$ counterpart, for example, in (spin-1/2) Heisenberg antiferromagnets (AFM): The (quasi-1D) ladders exhibit in the groundstate an odd-even effect, i.e., odd-leg ladders have one gapless spinon-mode, while even-leg ladders have a spin-gap [1]. The 2D Heisenberg model however, has two gapless magnon-modes and long-range order [2,3]. Similarly, (quasi-)1D conductors are generally Luttinger liquids (LL) 4, 4 , while 2D and 3D electron systems are (usually) Fermi liquids (FL).

For the AFM Heisenberg model, the dimensional crossover from 1D to 2D has been studied in Ref. [6]. Via the correspondence between number of legs $N$ and temperature $T, 1 / T \propto N$, the nonlinear sigma-model allows one in this case to calculate various physical properties; in particular, the spin-gap present in even-leg ladders vanishes exponentially as a function of the number $N$ of coupled spin-chains. Coupled LLs [7] and the dimensional crossover to $2 \mathrm{D}$ have been investigated by many different authors 8 10]. One of the reasons was the search for a non-FL (i.e., possible LL-like) phase in 2D 11]. However, no clear evidence for such a phase has been found so far [8 10,12].

In the half-filled and lightly doped Hubbard model, there is a strong competition between Cooper, umklapp, and AFM processes. For an unbiased determination of the dominant phase, it is therefore advantageous to use the renormalization group (RG) technique [13]. In the (weakly interacting) 2D case, such RG studies found AFM at and close to half-filling and $d$-wave superconductivity for sufficient hole doping [14 17]. The dimensional crossover from 1D to 2D was only investigated away from half-filling (without umklapp and AFM processes) [18]. The authors concluded that the system becomes a FL for $N \rightarrow \infty$.

In a previous work, we have studied in detail the groundstate properties of the half-filled and lightly doped $N=3$ and 4-leg Hubbard ladders in the case of weak interactions 19. In contrast to the large $U$ case, where the half-filled Hubbard model converges onto the Heisenberg model of spin-1/2 20, in the small $U$ case, the charge degrees of freedom are still present, which gives insight in the effect of doping away from half-filling.

Here, we analyze the crossover from $1 \mathrm{D}$ to $2 \mathrm{D}$ for the half-filled (and lightly doped) ladders. We will show that depending on the energy-scale (respectively length-scale), the physical properties are very different and that the phases associated with these properties are separated by crossover energies which are a function of $N$. In particular, the quasi-1D analog of the $2 \mathrm{D}$ phase is present at higher energies, implying that the crossover between different phases can be studied for $N$ finite. An advantage in quasi-1D compared to the $2 \mathrm{D}$ case is the limited number of different interactions. This allows us to obtain (some) analytical results and to disentangle the interplay between AFMs (two gapless magnon-modes), ISLs (insulating spin-liquid, i.e., only short-range correlations), and superconductivity. The $2 \mathrm{D}$ limit $(N=\infty)$ can then be taken within the same phase. 
For weak interactions, the Hubbard ladders are equivalent to a $N$-band model, characterized by Fermi velocities $v_{j}$. At half-filling, due to nesting of the Fermi surface (FS), the Fermi velocities of the bands $j$ and $N+1-j$ are equal (to be called "band pairs" below), such that AFM and umklapp processes take place. Using RG techniques, we find that the half-filled ladders exhibit (in the spin-sector) an odd-even effect only below a crossover energy $E_{c} \propto \exp [-\alpha \exp (\gamma N)](\alpha \ll 1$ and $\gamma \sim 1)$, but are 2 D-like (insulating) AFMs above the scale $E_{c}$. The reason is, that below $E_{c}$ only interactions within band pairs $(j, N+1-j)$ [and within the band $(N+1) / 2$ for $N$ odd] are present, while above $E_{c}$ all bands are interacting with each other. We obtain an analytical expression for the Hamiltonian which is similar to the 2D Heisenberg AFM. Analyzing the charge-sector by bosonization techniques [5.21.22], we find, that the Mott insulator is of the same type as in $N / 2$ half-filled two-leg ladders. The scale $E_{c}$ decreases fast to zero as the number of chains $N$ goes to infinity. The 2D limit is thus an insulating AFM, in agreement with what has been found in studies of the weakly interacting 2D Hubbard model 14 17.

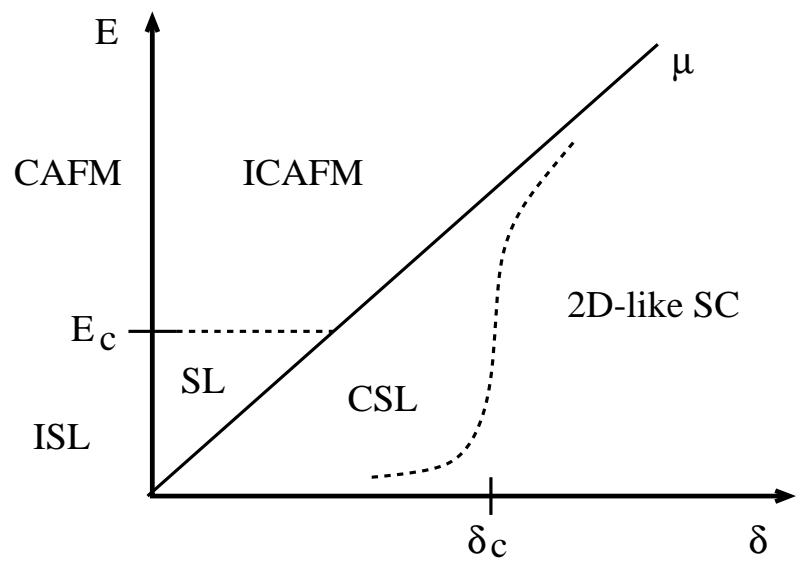

FIG. 1. Schematic phase diagram for the (large) $N$-leg Hubbard ladders. At half-filling, the system below the energy-scale $E_{c}=E_{c}(N)$ is an insulating spin-liquid (ISL) and above $E_{c}$ an insulating, 2D-like, commensurate antiferromagnet (CAFM). Upon doping away from half-filling, the ladders become below the chemical potential $\mu$ (measured from its value at half-filling) a conducting spin-liquid (CSL), i.e., phase coherence is only present within pairs of Fermi momenta $\left(k_{F}, \pi-k_{F}\right)$ and $\left(\pi-k_{F}, k_{F}\right)$; above $\mu$, the antiferromagnetic correlations become incommensurate (ICAFM). Upon increasing doping, phase coherence between all Fermi momenta sets in and the system becomes a 2D-like $d$-wave superconductor.

Doping away from half-filling (at energies above $E_{c}$ ), we find that the effect of a doping $\delta>0$ is the same as of a finite $N<\infty$ : Doped holes suppress the interactions between unpaired bands and thereby reduce the antiferromagnetic correlations. The effect of the doping on the remaining interactions within a band pair $(j, N+1-j)$ is then the same as when doping $N / 2$ uncoupled ISLs; there is a spin-gap and $d$-wave-like phase coherence between bands $j$ and $N+1-j$. At a higher doping value $\delta_{c}=\delta_{c}(N)$, phase coherence between all band pairs sets in and the $N$-leg ladders become a 2D-like $d$-wave superconductor $\left[\delta_{c}(N) \rightarrow 0\right.$ for $\left.N \rightarrow \infty\right]$. The low-energy physics is given by a $d$-wave BCS Hamiltonian. The origin of the superconducting instability is the same as for the 2D case 14 16,: A Kohn-Luttinger-type attraction [23] is generated by AFM processes [24]. Furthermore, while at half-filling the antiferromagnetic correlations are commensurate, upon doping, they become incommensurate. Fig. 1 gives a schematic overview.

Summarizing, the $N$-leg Hubbard model exhibits below the length-scale $\xi_{c} \propto 1 / E_{c}$ almost the same physical properties as the $2 \mathrm{D}$ model (i.e., the same correlation functions dominate), but in contrast to the $2 \mathrm{D}$ case, this quasi-1D analog can be treated (partially) analytically.

We do all our calculations for even $N$ and only refer to the odd $N$ case where it is of interest. In Sec. II, we introduce the $N$-leg ladder Hamiltonian, discuss the various interactions, and specify along which "path" the dimensional crossover is studied. In Sec. III, we briefly revisit the results obtained previously in Ref. [19] and analyze the half-filled Hamiltonian as a function of the energyscale. We show that there exist for finite $N$ two clearly different phases, separated by a crossover energy $E_{c}$. We derive an (analytical) expression for $E_{c}$. In Sec. IV, the phase above the energy $E_{c}$ is investigated in detail and shown to be a 2D-like AFM. Finally, in Sec. V, we treat the effect of doping away from half-filling.

\section{II. $N$-LEG HUBBARD MODEL}

The $N$-leg ( $N$-chain) Hubbard model is given by $H=$ $H_{0}+H_{\text {Int }}$, where the kinetic energy is

$$
\begin{array}{r}
H_{0}=-t \sum_{x, i, s} d_{i s}^{\dagger}(x+1) d_{i s}(x)+\text { H.c. } \\
-t_{\perp} \sum_{x, i, s} d_{i+1 s}^{\dagger}(x) d_{i s}(x)+\text { H.c. }
\end{array}
$$

and the interaction term is

$$
H_{\mathrm{Int}}=U \sum_{i, x} d_{i \uparrow}^{\dagger}(x) d_{i \uparrow}(x) d_{i \downarrow}^{\dagger}(x) d_{i \downarrow}(x) .
$$

The hopping matrix elements along- and perpendicular to the chains are denoted by $t$ and $t_{\perp}$ respectively and $U>0$ is the on-site repulsion. For our approach, it is advantageous first to diagonalize $H_{0}$,

$$
H_{0}=\sum_{j, s} \int d k \epsilon_{j}(k) \Psi_{j s}^{\dagger}(k) \Psi_{j s}(k),
$$

where $\Psi_{j s}^{\dagger}$ and $\Psi_{j s}$ are the creation- and annihilation operators for the band $j$. For open boundary conditions 
perpendicular to the legs, the dispersion relations $\epsilon_{j}$ are given by $(j=1, \ldots, N)$

$$
\epsilon_{j}(k)=-2 t \cos (k)-2 t_{\perp} \cos [\pi j /(N+1)] .
$$

The Fermi momenta in each band $k_{F j}$ are determined by the chemical potential $\mu=\epsilon_{j}\left(k_{F j}\right)$ and the filling $n=2(\pi N)^{-1} \sum k_{F j}$. Since we are only interested in the low-energy physics, we linearize the dispersion relation at the FS, resulting in Fermi velocities $v_{j}=2 t \sin \left(k_{F j}\right)$. We introduce operators $\Psi_{R / L j s}$ for right and left movers at the FS. At half-filling, $\mu=0$, and

$$
v_{j}=v_{\bar{\jmath}}=2 \sqrt{t^{2}-t_{\perp}^{2} \cos [\pi j /(N+1)]^{2}},
$$

where $\bar{\jmath}=N+1-j$. For $t=t_{\perp}, v_{1}=v_{N} \approx 2 \pi t / N$, which leads to a singular behavior for large $N$ (the quasi$1 \mathrm{D}$ analog of the van Hove singularities in 2D). For the following, we take $t_{\perp}<t$ in order to avoid singularities. Note that the FS is then not flat 25], but that we still have nesting, i.e., $k_{F j}+k_{F \bar{\jmath}}=\pi$.

The crucial difference between quasi-1D and 2D are the interactions which control the low-energy physics. The system is quasi-1D and only a finite number of different interactions play a role, provided the energy difference between two neighboring bands is larger than the largest energy-scale of the system, i.e., $t e^{-t / U}$. Therefore, we investigate the crossover between different phases (ISL and AFM) in quasi-1D, $U \ll t / \ln N, t_{\perp} / \ln N$, as a function of the energy (this can partially be done analytically). The 2D limit, $\ln N \gg t / U$, is then taken within the same phase. In other words, along this particular "path", the dimensional crossover from $1 \mathrm{D}$ to $2 \mathrm{D}$ can be treated in a controlled way.

Since we are interested in the small- $U$ low-energy physics, we have to take into account only the processes, which are present down to arbitrarily small energy-scales. For the half-filled case, this implies that only umklapp processes where the momenta add up exactly to $\pi$, $k_{F l}+k_{F m}=\pi$ are relevant. This condition is only fulfilled for the band pairs $(j, \bar{\jmath}), k_{F j}+k_{F \bar{\jmath}}=\pi$. When doping away from half-filling, the chemical potential (measured from its value at half-filling) introduces a lowenergy cutoff for the various umklapp processes. The interactions which have to be taken into account initially are then different.

At half-filling, the interacting part of the Hamiltonian consists of forward (f), Cooper (c), and umklapp (u) processes between 2 different bands and (non-)umklapp processes between 4 different bands (in our notation, we follow Refs. 19.26), $H_{\text {Int }}=H^{2 B}+H^{4 B}$, where

$$
\begin{aligned}
H^{2 B}= & \sum_{i \neq j} \int d x\left(f_{i j}^{\rho} J_{R i i} J_{L j j}-f_{i j}^{\sigma} \mathbf{J}_{R i i} \cdot \mathbf{J}_{L j j}\right. \\
& \left.+c_{i j}^{\rho} J_{R i j} J_{L i j}-c_{i j}^{\sigma} \mathbf{J}_{R i j} \cdot \mathbf{J}_{L i j}\right) \\
& +\sum_{j} \int d x\left(c_{j j}^{\rho} J_{R j j} J_{L j j}-c_{j j}^{\sigma} \mathbf{J}_{R j j} \cdot \mathbf{J}_{L j j}\right)
\end{aligned}
$$

$$
\begin{aligned}
& +\sum_{j} \int d x\left[\left(u_{j \bar{j} \bar{\jmath} j}^{\rho} I_{R j \bar{\jmath}}^{\dagger} I_{L \bar{\jmath} j}-u_{j \bar{\jmath} \bar{j}}^{\sigma} \mathbf{I}_{R j \bar{\jmath}}^{\dagger} \cdot \mathbf{I}_{L \bar{\jmath} j}\right.\right. \\
& \left.\left.+u_{j j \bar{\jmath} \bar{\jmath}}^{\rho} I_{R j j}^{\dagger} I_{L \bar{\jmath} \bar{\jmath}}\right)+ \text { H.c. }\right]
\end{aligned}
$$

where the $\mathrm{U}(1)$ and $\mathrm{SU}(2)$ currents are defined as

$$
J_{h i j}=\sum_{s} \Psi_{h i s}^{\dagger} \Psi_{h j s}
$$

and

$$
J_{h i j}^{p}=\frac{1}{2} \sum_{s, s^{\prime}} \Psi_{h i s}^{\dagger} \tau_{s s^{\prime}}^{p} \Psi_{h j s^{\prime}}
$$

where again $h=R / L$ and the $\tau^{p}$ are the Pauli matrices $(p=x, y, z)$. Due to symmetry, $f_{i j}^{\rho, \sigma}=f_{j i}^{\rho, \sigma}$ and $c_{i j}^{\rho, \sigma}=c_{j i}^{\rho, \sigma}$. The umklapp currents are

$$
I_{h i j}=\sum_{s, s^{\prime}} \Psi_{h i s} \epsilon_{s s^{\prime}} \Psi_{h j s^{\prime}}
$$

and

$$
I_{h i j}^{p}=\frac{1}{2} \sum_{s, s^{\prime}} \Psi_{h i s}\left(\epsilon \tau^{p}\right)_{s s^{\prime}} \Psi_{h j s^{\prime}}
$$

where $\epsilon=-i \tau^{y}$. The 4-band interactions involve processes between two different band pairs $(j, \bar{\jmath})$ and $(k, \bar{k})$,

$$
H^{4 B}=\sum_{j=1}^{N / 2-1} \sum_{k=j+1}^{N-j} \int d x \mathcal{H}_{j k}^{4 B},
$$

where

$$
\begin{array}{r}
\mathcal{H}_{j k}^{4 B}=c_{j k \bar{k} \bar{\jmath}}^{\rho}\left(J_{R j k} J_{L \bar{k} \bar{\jmath}}+J_{R \bar{j} \bar{k}} J_{L k j}+\text { H.c. }\right) \\
-c_{j k \bar{k} \bar{\jmath}}^{\sigma}\left(\mathbf{J}_{R j k} \cdot \mathbf{J}_{L \bar{k} \bar{\jmath}}+\mathbf{J}_{R \bar{\jmath} \bar{k}} \cdot \mathbf{J}_{L k j}+\text { H.c. }\right) \\
+u_{j k \bar{k} \bar{\jmath}}^{\rho}\left(I_{R j k}^{\dagger} I_{L \bar{k} \bar{\jmath}}+I_{R \bar{k} \bar{\jmath}}^{\dagger} I_{L j k}+\text { H.c. }\right) \\
+u_{j \bar{j} k \bar{k}}^{\rho}\left(I_{R j \bar{j}}^{\dagger} I_{L k \bar{k}}+I_{R k \bar{k}}^{\dagger} I_{L j \bar{\jmath}}+\text { H.c. }\right) \\
-u_{j k \bar{k} \bar{\jmath}}^{\sigma}\left(\mathbf{I}_{R j k}^{\dagger} \cdot \mathbf{I}_{L \bar{k} \bar{\jmath}}+\mathbf{I}_{R \bar{k} \bar{\jmath}}^{\dagger} \cdot \mathbf{I}_{L j k}+\text { H.c. }\right) .
\end{array}
$$

In our case $v_{j}=v_{\bar{\jmath}}$ implying $c_{j k \bar{k} \bar{\jmath}}^{\rho, \sigma}=c_{j \bar{k} k \bar{\jmath}}^{\rho, \sigma}, u_{j k \bar{k} \bar{\jmath}}^{\rho, \sigma}=u_{j \bar{k} k \bar{\jmath}}^{\rho, \sigma}$. Note that the 4-band couplings describe AFM processes, i.e., the terms $\Psi_{R j s}^{\dagger} \Psi_{L \bar{\jmath} s^{\prime}}$ result in a momentum transfer of $\pi$ (along the chains).

\section{DIMENSIONAL CROSSOVER}

Using the RG method, we study the half-filled $N$-leg ladders as a function of the energy-scale. We show that there exist two clearly different phases, separated by a crossover energy $E_{c} \ll t$. The phase below $E_{c}$ is an 
ISL, i.e., 4-band-AFM processes are suppressed and the physics is dominated by 2-band umklapp and Cooper processes [19]. In contrast, in the phase above $E_{c}$ (but still below the bandwidth $t$ ) the 4-band-AFM processes are large and dominating (this phase is investigated in detail in Sec. IV). First (Sec. III A), we revisit the results for $N$-leg ladders obtained previously in Ref. [19] and give a criterion for the couplings allowing for a (rather) precise distinction between the two phases. In Sec. III B, we calculate the crossover energy $E_{c}$ as a function of $N$.

\section{A. ISL phase in even-leg ladders}

In Ref. 19], we have shown, that at half-filling (for $N$ even) the band pair $(j, \bar{\jmath})$ scales towards an ISL and is frozen out at the energy-scale $t e^{-\alpha_{j} v_{j} / U}$, where $\alpha_{j} \sim 1$. The low-energy Hamiltonian is then the same as of $N / 2$ half-filled two-leg ladders. For increasing $N$, the initialvalue $U / t$ (and therefore the energy-scale), which leads to this decoupling (in particular to a suppression of the 4band-AFM processes) and an ISL phase, decreases fast. Next, using bosonization techniques [5.21,22], we show that the RG flow of the Luttinger liquid parameter for spin triplet pairing, $K_{\sigma j+}$, allows one to determine the energy-scale, where the spin-gap opens. This energyscale can then be interpreted as the crossover energy between the ISL phase at low energies and the AFM phase at higher energies (which has no spin-gap).

Bosonization is the method of rewriting fermionic creation (annihilation) operators in terms of field operators $\Phi_{\alpha}$ and $\Pi_{\alpha}$ satisfying the commutation relation $\left[\Phi_{\alpha}(x), \Pi_{\alpha}(y)\right]=i \delta(x-y)(\alpha$ labels the charge and spin-modes for the different bands). The dual field of $\Phi_{\alpha}$ is defined as $\partial_{x} \theta_{\alpha}=\Pi_{\alpha}$. The fields $\Phi_{\alpha}$ contain the (physical) charge respectively spin of the particle, i.e., the charge/spin-density is $\rho_{\alpha}=\partial_{x} \Phi_{\alpha}$, while the fields $\theta_{\alpha}$ can be interpreted as the phase of the chargepart respectively the spin-part (for ladders, see, e.g., Refs. [18, 19,26]). We introduce the fields

$$
\Phi_{\nu j \bar{\jmath} \pm}=\frac{1}{\sqrt{2}}\left(\Phi_{\nu j} \pm \Phi_{\nu \bar{\jmath}}\right)
$$

and

$$
\Pi_{\nu j \bar{\jmath} \pm}=\frac{1}{\sqrt{2}}\left(\Pi_{\nu j} \pm \Pi_{\nu \bar{\jmath}}\right)
$$

combining the fields of the bands $j$ and $\bar{\jmath}(\nu=\rho, \sigma)$, e.g., the spin-fields $\Phi_{\sigma j \bar{\jmath} \pm}$ describe triplet/singlet pairs between the momenta $k_{F j}$ and $k_{F \bar{\jmath}}$.

The bosonized Hamiltonian takes the following form. The noninteracting Hamiltonian $H_{0}$ plus the contributions of the charge-currents $f_{j \bar{j}}^{\rho}$ and $c_{j j}^{\rho}$ and the zcomponent of the spin-currents $f_{j \bar{\jmath}}^{\sigma}$ and $c_{j j}^{\sigma}$ can be written as

$$
\tilde{H}_{0}=\sum_{j, \nu, \mu} \int d x \frac{v_{\nu j \mu}}{2}\left[\frac{1}{K_{\nu j \mu}}\left(\partial_{x} \Phi_{\nu j \bar{\jmath} \mu}\right)^{2}+K_{\nu j \mu} \Pi_{\nu j \bar{\jmath} \mu}^{2}\right],
$$

where $\nu=\rho, \sigma$ and $\mu= \pm$ and the Luttinger liquid parameters (LLP) are

$$
K_{\rho j \pm}=\sqrt{\frac{\pi v_{j}-\left(c_{j j}^{\rho} \pm f_{j \bar{j}}^{\rho}\right)}{\pi v_{j}+\left(c_{j j}^{\rho} \pm f_{j \bar{j}}^{\rho}\right)}}
$$

and

$$
K_{\sigma j \pm}=\sqrt{\frac{4 \pi v_{j}+\left(c_{j j}^{\sigma} \pm f_{j \bar{j}}^{\sigma}\right)}{4 \pi v_{j}-\left(c_{j j}^{\sigma} \pm f_{j \bar{j}}^{\sigma}\right)}}
$$

The velocities are $v_{\nu j \mu} \sim v_{j}$. The interacting part of the $N$-leg ladder Hamiltonian contains (apart form other terms) the expression [26]

$$
-\cos \left(\sqrt{4 \pi} \Phi_{\sigma j \bar{\jmath}+}\right) \times \text { other cosine terms. }
$$

Fields which appear in a cosine become "pinned" in order to minimize the energy, e.g., the term $-\cos \left(\sqrt{4 \pi} \Phi_{\sigma j \bar{\jmath}+}\right)$ implies (quasiclassicaly) $\Phi_{\sigma j \bar{j}+} \approx 0$ and the corresponding spin-mode acquires a gap.

The LLPs determine the exponents of correlation functions 21 22] and - together with cosine-terms - the pinned fields. Here, as we show in the following, the flow of the LLP $K_{\sigma j+}$ can be used to determine the energy-scale where the spin-gap in the $\sigma+$ sector opens. Note that initially (i.e., at high energies) $K_{\sigma j+}>1$ and $K_{\sigma j-}=1$ (and $K_{\rho j+}<1, K_{\rho j-}=1$ ). For the $N$-leg ladders, the RG flow of the couplings is such that $c_{j j}^{\sigma} \approx-t$ and $f_{j \bar{j}}^{\sigma} \approx 0$ at low energies, implying $K_{\sigma j \pm}<1$. Hence, while $K_{\sigma j-}$ is always $\leq 1$, there is an energy, where $K_{\sigma j+}$ becomes smaller than 1 , i.e., $r_{j}=c_{j j}^{\sigma}+f_{j \bar{\jmath}}^{\sigma} \leq 0$. The canonical transformation

$$
\tilde{\Phi}_{\sigma j \bar{\jmath}+}=\Phi_{\sigma j \bar{\jmath}+} / \sqrt{K_{\sigma j+}}, \quad \tilde{\theta}_{\sigma j \bar{\jmath}+}=\theta_{\sigma j \bar{\jmath}+} \sqrt{K_{\sigma j+}}
$$

eliminates $K_{\sigma j+}$ from $\tilde{H}_{0}$ (it just renormalizes the velocities) and results in

$$
-\cos \left(\sqrt{4 \pi K_{\sigma j+}} \tilde{\Phi}_{\sigma j \bar{\jmath}+}\right) \times \text { other cosine terms. }
$$

A large $K_{\sigma j+}$ therefore tends to depin the field $\tilde{\Phi}_{\sigma j \bar{\jmath}+}$, while a small $K_{\sigma j+}$ results in a strong pinning of the field $\tilde{\Phi}_{\sigma j \bar{\jmath}+}$ (for the dual field $\tilde{\theta}_{\sigma j \bar{\jmath}+}$, it is vice versa). The crossover between the two regimes takes place at $K_{\sigma j+}=1$ (since we are at finite energy, it is a crossover and not a transition). The ISL phase therefore requires $r_{j}<0\left(K_{\sigma j+}<1\right)$ and $r_{j}$ can be used in the RG flow to determine a crossover energy. We note that for the pure sine-Gordon model (at zero temperature), the value $K_{\alpha}=1$ rigorously separates the massive from the massless phase [27. 

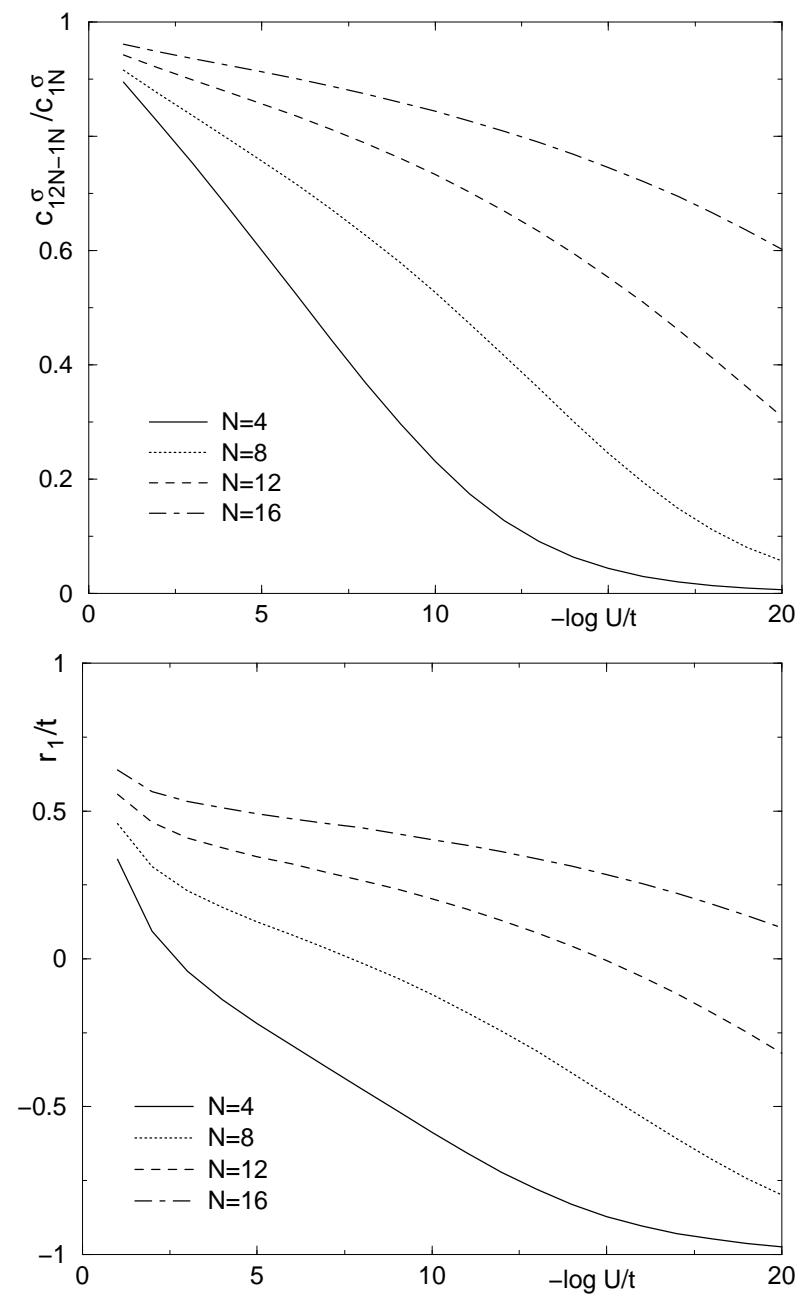

FIG. 2. The upper figure shows the strong-coupling value of the ratio of a 4-band-AFM and a 2-band Cooper coupling, $c_{12 N-1 N}^{\sigma} / c_{1 N}^{\sigma}$, as a function of the initial-value $U / t$. For increasing $N$, the 4-band-AFM couplings are only suppressed for very small initial-values. Similarly, the lower figure displays the strong-coupling value of the sum of couplings $r_{1}=c_{11}^{\sigma}+f_{1 N}^{\sigma}$. The initial-values which lead to $r_{1}<0$ (i.e., an ISL) become very small as $N$ becomes large. We take both times $t_{\perp} / t=0.95$.

\section{B. Determination of the crossover energy}

Performing a numerical integration of the full set of RG equations (RGEs) [28], we calculate the crossover energy $E_{c}=E_{c}(N)$, which separates ladder-like from 2D-AFMlike behavior (the RGEs are a generalization of the 3-leg RGEs given in Ref. [19]; the RGEs relevant for the AFM phase are given below).

In Fig. 2, we have plotted the strong-coupling value of the ratio of a 4-band AFM and a 2-band Cooper coupling, $c_{12 N-1 N}^{\sigma} / c_{1 N}^{\sigma}$, and of $r_{1}=c_{11}^{\sigma}+f_{1 N}^{\sigma}$ for $N=4,8,12,16$ (we fix the strong-coupling value of $c_{1 N}^{\sigma}$ at the bandwidth $t$ ). We take $t_{\perp} / t=0.95$, because for $t_{\perp} / t=1$, the band pair $(1, N)$ is close to the van Hove singularities, such that its behavior would no more be typical for the system. When the number of chains, $N$, increases, the initial-value $U / t$ which leads to a suppression of the 4band-AFM couplings decreases fast. Similarly, for large $N$, the $r_{1}$ becomes only negative when $U / t$ is very small. Note that for the other band pairs $(j, \bar{j})$, the corresponding energy-scales are lower, since the velocities $v_{j}$ are larger and the gaps are of the order of $t \exp \left(-\alpha_{j} v_{j} / U\right)$.

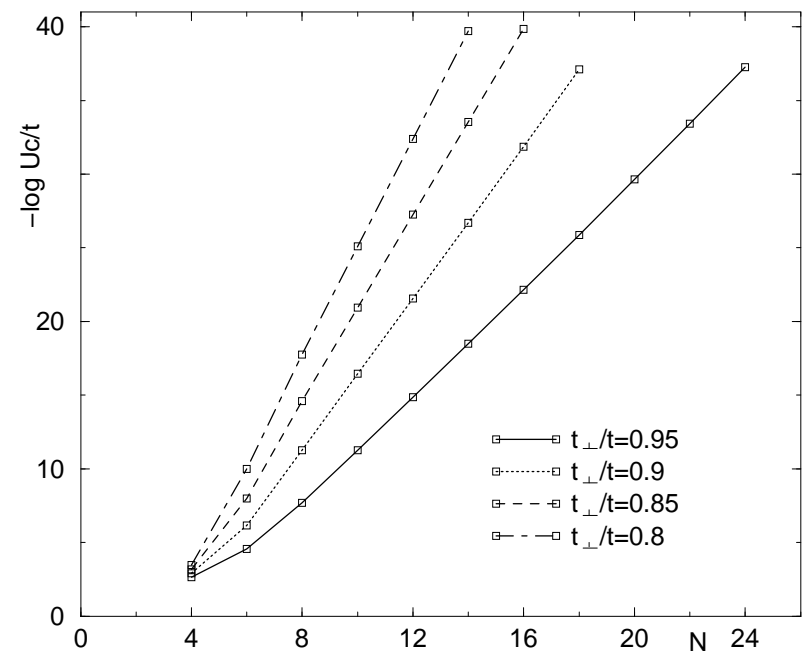

FIG. 3. The sum of couplings $r_{j}=c_{j j}^{\sigma}+f_{j \bar{\jmath}}^{\sigma}$ allows to determine between ISL and AFM: For $r_{j}<0$ the band pair $(j, \bar{\jmath})$ is an ISL and for $r_{j}>0$ an AFM. We have plotted the initial-value $U_{c} / t$, which leads to $r_{1} \approx 0$ at the energy-scale, where the first coupling of the band pair $(1, N)$ has grown up to the bandwidth $t$. The figure shows, that $-\log U_{c}(N) / t$ is in good approximation a linear function of $N$ (the squares are the calculated initial-values and the lines are a guide to the eye).

Hence, both the 4-band-AFM processes and the $r_{j}$ have a strong and similar dependence on the energy-scale. For small enough initial-values $U / t$, the differences between the Fermi velocities lead to a decoupling into band pairs. However, since $v_{j}-v_{j+1} \propto 1 / N$, for increasing $N$, this decoupling becomes suppressed and the physics is dominated by 4 -band-AFM processes.

We calculate the crossover energy $E_{c}$ as follows. We determine initial-values $U_{c}=U_{c}(N)$ such that $r_{1} \approx 0$ at the scale where the first coupling of the band pair $(1, N)$ has grown up to the bandwidth $t$. For initialvalues $U<U_{c}(N)$, a spin-gap then opens, starting in the band pair $(1, N)$, and the 4-band-AFM processes become suppressed. We find that $U_{c}(N)$ can be fitted by $U_{c}(0) \exp (-\gamma N)$, where $\gamma \sim 1$ (for $t_{\perp} / t=$ $0.8,0.85,0.9,0.95$, we obtain $\gamma=3.7,3.2,2.6,1.9)$ and $U_{c}(0) \gg t$, see Fig. 3. On the other hand, an initial-value $U$ corresponds to an energy-scale (gap) $t \exp (-t / U)$. The crossover energy $E_{c}$ is therefore given by

$$
E_{c} \sim t \exp [-\alpha \exp (\gamma N)]
$$


where $\alpha \sim t / U_{c}(0) \ll 1$. Note that the energy $E_{c}$ is also the upper limit for the spin-gap in the ISL phase. For large $U$ (Heisenberg AFM), the corresponding scale (spin-gap) is $J \exp (-0.68 N)$, where $J=4 t^{2} / U$ (see also table I) [6]. While for large $U$ the spin-gap decreases exponentially, in the small- $U$ case the decrease is doubleexponentially.

As a result, the "phase" of the $2 \mathrm{D}$ system is for finite $N$ present above an energy-scale $E_{c}=E_{c}(N)$, where $E_{c}(N) \rightarrow 0$ for $N \rightarrow \infty$. Equivalently, below the lengthscale $\xi_{c} \propto 1 / E_{c}$, the dominating correlation functions are the same as in the $2 \mathrm{D}$ system.

\section{THE 2D-LIKE AFM PHASE}

First (Sec. IV A), using the RG method, we calculate the asymptotic ratios of the couplings and the chargegap of the phase present above $E_{c}$. Comparing our results with the flow well away from half-filling [18], we conclude, that AFM processes (at high energies) are necessary for an instability in the RG flow of the $N$-leg Hubbard model. In Sec. IV B, we show that the phase above $E_{c}$ is the quasi-1D counterpart of a $2 \mathrm{D}$ insulating AFM. In particular, there is no odd-even effect and the interacting part of the Hamiltonian is similar to the 2D Heisenberg AFM. Using bosonization techniques, we obtain, that the system is Mott insulating and that the insulator is of the same type as in the half-filled twoleg ladder, see Sec. IV C. For the spin-sector, we find the same physical properties as for the Heisenberg AFM, i.e., two gapless magnon modes, spinon confinement, and long-range order in the $2 \mathrm{D}$ limit.

\section{A. Asymptotic ratios of the couplings}

We give the RGEs of the 4-band-AFM couplings for large $N$, i.e., keeping in the RGEs only sums over $N$ products of couplings and dropping the (2-band) contributions of the order of 1 . We then determine (analytically) the asymptotic ratios of the couplings for Hubbard initial-values and the size of the charge-gap.

\section{RGEs}

The RGEs of the 4-band interactions are given by a sum over $N / 2$ products of 4 -band couplings

$$
\begin{aligned}
\frac{d c_{j k \bar{k} \bar{\jmath}}^{\rho}}{d l}= & \sum_{i \neq j, k}^{N / 2} \frac{1}{v_{i}}\left(c_{j i \bar{\imath} \bar{\jmath}}^{\rho} c_{k i \bar{\imath} \bar{k}}^{\rho}+u_{j i \bar{\imath} \bar{\jmath}}^{\rho} u_{k i \bar{\imath} \bar{k}}^{\rho}\right. \\
& \left.+\frac{3}{16} c_{j i \bar{\imath} \bar{\jmath}}^{\sigma} c_{k i \bar{\imath} \bar{k}}^{\sigma}+\frac{3}{16} u_{j i \bar{\imath} \bar{\jmath}}^{\sigma} u_{k i \bar{\imath} \bar{k}}^{\sigma}\right)
\end{aligned}
$$

$$
\begin{aligned}
& \frac{d u_{j k \bar{k} \bar{\jmath}}^{\rho}=}{d l} \sum_{i \neq j, k}^{N / 2} \frac{1}{v_{i}}\left(c_{j i \bar{\imath} \bar{\jmath}}^{\rho} u_{k i \bar{\imath} \bar{k}}^{\rho}-\frac{3}{16} c_{j i \bar{\imath} \bar{\jmath}}^{\sigma} u_{k i \bar{\imath} \bar{k}}^{\sigma}+k \leftrightarrow j\right) \\
& \frac{d c_{j k \bar{k} \bar{\jmath}}^{\sigma}=}{d l}=\sum_{i \neq j, k}^{N / 2} \frac{1}{v_{i}}\left(-\frac{1}{2} c_{j i \bar{\imath} \bar{\jmath}}^{\sigma} c_{k i \bar{\imath} \bar{k}}^{\sigma}+c_{j i \bar{\imath} \bar{\jmath}}^{\sigma} c_{k i \bar{\imath} \bar{k}}^{\rho}+c_{k i \bar{\imath} \bar{k}}^{\sigma} c_{j i \bar{\imath} \bar{\jmath}}^{\rho}\right. \\
&\left.-\frac{1}{2} u_{j i \bar{\imath} \bar{\jmath}}^{\sigma} u_{k i \bar{\imath} \bar{k}}^{\sigma}-u_{j i \bar{\imath} \bar{\jmath}}^{\sigma} u_{k i \bar{\imath} \bar{k}}^{\rho}-u_{k i \bar{\imath} \bar{k}}^{\sigma} u_{j i \bar{\imath} \bar{\jmath}}^{\rho}\right) \\
& \frac{d u_{j k \bar{k} \bar{\jmath}}^{\sigma}=}{d l} \sum_{i \neq j, k}^{N / 2} \frac{1}{v_{i}}\left(-\frac{1}{2} c_{j i \bar{\imath} \bar{\jmath}}^{\sigma} u_{k i \bar{\imath} \bar{k}}^{\sigma}+u_{j i \bar{\imath} \bar{\jmath}}^{\sigma} c_{k i \bar{\imath} \bar{k}}^{\rho}\right. \\
&\left.-u_{j i \bar{\imath} \bar{\jmath}}^{\rho} c_{k i \bar{\imath} \bar{k}}^{\sigma}+k \leftrightarrow j\right)
\end{aligned}
$$

where the energy-scale is related to $l$ by $E \sim t e^{-\pi l}$. Defining

$$
h_{j k}^{ \pm}=\frac{c_{j k \bar{k} \bar{j}}^{\sigma}}{4}+\frac{u_{j k \bar{k} \bar{j}}^{\sigma}}{4} \pm\left(c_{j k \bar{k} \bar{\jmath}}^{\rho}-u_{j k \bar{k} \bar{\jmath}}^{\rho}\right)
$$

we find from the above RGEs, that

$$
\frac{d}{d l} h_{j k}^{+}=\sum_{i \neq j, k}^{N / 2} \frac{1}{v_{i}} h_{j i}^{+} h_{k i}^{+} .
$$

Given the Hubbard initial-values $4 c_{j k \bar{k} \bar{\jmath}}^{\rho}=c_{j k \bar{k} \bar{\jmath}}^{\sigma}=$ $2 u_{j k \bar{k} \bar{\jmath}}^{\rho}=2 U /(N+1)$ and $u_{j k \bar{k} \bar{j}}^{\sigma}=0$, we obtain $h_{j k}^{+}(l)=0$ for all $l$ (this fixed point is stabilized by the 2-band interactions). Since

$\frac{d}{d l} h_{j k}^{-}=-\sum_{i \neq j, k} \frac{1}{v_{i}}\left[h_{j i}^{-} h_{k i}^{-}+\left(h_{j i}^{+}+h_{j i}^{-}\right)\left(h_{k i}^{+}+h_{k i}^{-}\right)\right]$

and $h_{j k}^{-}(0)>0$, the $h_{j k}^{-}$flow to $0, h_{j k}^{-}(l) \rightarrow 0$ for increasing $l$. From the flow of the $h_{j k}^{ \pm}$, we then calculate the flow of the 4-band couplings and obtain the asymptotic ratios

$$
t \sim 3 g_{j k}=4 c_{j k \bar{k} \bar{\jmath}}^{\rho}=3 c_{j k \bar{k} \bar{\jmath}}^{\sigma}=4 u_{j k \bar{k} \bar{\jmath}}^{\rho}=-3 u_{j k \bar{k} \bar{\jmath}}^{\sigma} .
$$

TABLE I. The table shows the correspondence between crossover energy (spin-gap) and energy-scale of the 2D system for small and large $U$. The exponentially small charge-gap for small $U$ leads to a double exponentially suppressed 1D-2D crossover energy (the result for the spin-gap in the large $U$ case is from Ref. [6]).

\begin{tabular}{lcc}
\hline \hline & 2D energy-scale & crossover energy \\
\hline$U \ll t$ & $t \exp (-\lambda t / U)$ & $t \exp [-\alpha \exp (\gamma N)]$ \\
$U \gg t$ & $J$ & $J \exp (-0.68 N)$ \\
\hline \hline
\end{tabular}


Note that $u_{j \bar{\jmath} k \bar{k}}^{\rho}$ remains always small. The 2-band and single-band interactions are for large $N$ dominantly renormalized by the 4 -band interactions. The asymptotic ratios of the 2-band and single-band couplings can therefore be calculated by inserting the ratios (26) in the RGEs for these couplings [19]. We then find, that the following couplings of the band pairs $(j, \bar{\jmath})$ grow and approach fixed ratios:

$$
\begin{aligned}
t \sim 3 g_{j} & =4 f_{j \bar{\jmath}}^{\rho}=3 f_{j \bar{\jmath}}^{\sigma}=4 c_{j \bar{\jmath}}^{\rho}=3 c_{j \bar{\jmath}}^{\sigma} \\
& =4 u_{j \bar{\jmath} \bar{\jmath} j}^{\rho}=8 u_{j j \bar{\jmath} \bar{\jmath}}^{\rho}=3 u_{j \bar{\jmath} \bar{\jmath} j}^{\sigma} .
\end{aligned}
$$

The other 2-band and single-band couplings stay small; in particular the single-band $\mathrm{SU}(2)$ processes are small, $\left|c_{j j}^{\sigma}\right| \ll t$, but they have become attractive, $c_{j j}^{\sigma}<0$.

For bands $k$ and $j$, which are close together on the FS, $k \rightarrow j$, the 4 -band coupling becomes the same as the corresponding 2-band couplings, $g_{j k} \approx g_{j} \approx g_{k}$. In the limit $N \rightarrow \infty$, all the (2 and 4-band) $g$-couplings take the same value, $g_{j}=g_{m}=g_{k l}$. The gap $\Delta$ is then similarly calculated as the asymptotic ratios; using that

$$
\frac{d}{d l} s_{j k}=\sum_{i \neq j, k}^{N / 2} \frac{1}{v_{i}} s_{j i} s_{k i}
$$

where

$$
s_{j k}=\frac{c_{j k \bar{k} \bar{\jmath}}^{\sigma}}{4}-\frac{u_{j k \bar{k} \bar{\jmath}}^{\sigma}}{4}+c_{j k \bar{k} \bar{\jmath}}^{\rho}+u_{j k \bar{k} \bar{\jmath}}^{\rho}
$$

we find for the scale of divergence

$$
l_{c}=\frac{N+1}{U \sum_{j}^{N} 1 / v_{j}} .
$$

In particular, $l_{c}=2 t / U$ for $t_{\perp} / t=0$ and

$$
l_{c}=\frac{\pi t}{U} \frac{1}{\ln (2 N / \pi)}
$$

for $t=t_{\perp}$, where $\ln N<t / U$ for the validity of our calculations. The logarithmic corrections come from the fact that $v_{1}=v_{N} \sim t / N$ (van Hove singularities). The gap becomes

$$
\Delta \sim t e^{-l_{c}}=t e^{-\lambda t / U}
$$

where $\lambda$ is a function of $t_{\perp} / t$ and of the order of $1, \lambda \sim 1$ (see also table I).

\section{Discussion}

It is instructive to compare our result with the situation well away from half-filling, where the umklapp interactions can be neglected. In Ref. [18, it has been shown, that without umklapp interactions and for repulsive interactions, $U>0$, the large $N$ limit is a FL, i.e., the typical gap-size scales exponentially to $0, \propto e^{-N}$. The reason is, that the forward scattering (f) processes, which tend to drive the system towards a RG instability give contributions of the order of 1 , while the Cooper processes (c), which drive the system towards a FL, have a weight $\propto N$.

At half-filling, the situation is completely different: Here, the 4-band AFM processes with a weight $\propto N$ are (entirely) responsible for the $\mathrm{RG}$ instability at the energy-scale $\Delta \sim t e^{-\lambda t / U}$. Therefore, the $N$-leg Hubbard model exhibits for large $N$ only a RG instability, when 4band AFM processes are (at least initially) present. The van Hove singularities, see Eq. (31), lead to a further increase of the gap-size (but are not the reason for the instability).

\section{B. Effective low-energy Hamiltonian}

We show that above $E_{c}$, the Hamiltonian of the halffilled $N$-leg Hubbard ladders is similar to the 2D Heisenberg AFM.

At low energies (but above $E_{c}$ ), the ratios of the couplings are given by Eqs. (26) and (27), such that the U(1) and $\mathrm{SU}(2)$ 4-band interactions, see Eq. (12), simplify to

$$
\begin{gathered}
3 J_{R j k} J_{L \bar{k} \bar{\jmath}}-4 \mathbf{J}_{R j k} \cdot \mathbf{J}_{L \bar{k} \bar{\jmath}}=2 \Psi_{R j s}^{\dagger} \Psi_{L \bar{\jmath} s} \Psi_{L \bar{k} \bar{s}}^{\dagger} \Psi_{R k \bar{s}} \\
-2 \Psi_{R j s}^{\dagger} \Psi_{L \bar{\jmath} s} \Psi_{L \bar{k} s}^{\dagger} \Psi_{R k s}-4 \Psi_{R j s}^{\dagger} \Psi_{L \bar{\jmath} \bar{s}} \Psi_{L \bar{k} \bar{s}}^{\dagger} \Psi_{R k s}
\end{gathered}
$$

(for $s=\uparrow, \bar{s}=\downarrow$ and vice versa) and

$$
\begin{aligned}
& 3 I_{R j k}^{\dagger} I_{L \bar{k} \bar{\jmath}}+4 \mathbf{I}_{R j k}^{\dagger} \cdot \mathbf{I}_{L \bar{k} \bar{\jmath}}=2 \Psi_{R j s}^{\dagger} \Psi_{L \bar{\jmath} s} \Psi_{R k \bar{s}}^{\dagger} \Psi_{L \bar{k} \bar{s}} \\
& -2 \Psi_{R j s}^{\dagger} \Psi_{L \bar{\jmath} s} \Psi_{R k s}^{\dagger} \Psi_{L \bar{k} s}-4 \Psi_{R j s}^{\dagger} \Psi_{L \bar{\jmath} \bar{s}} \Psi_{R k \bar{s}}^{\dagger} \Psi_{L \bar{k} s} .
\end{aligned}
$$

Defining

$$
M_{j}^{p}=\Psi_{R j s}^{\dagger} \tau_{s s^{\prime}}^{p} \Psi_{L \bar{\jmath} s^{\prime}}+\text { H.c. }
$$

the Hamiltonian then takes the form

$$
H=H_{0}-\frac{1}{2} \sum_{i, j} g_{i j} \int d x \mathbf{M}_{i} \cdot \mathbf{M}_{j} .
$$

The 2-band couplings $f_{j \bar{j}}^{\rho, \sigma}$ and $u_{j j \bar{\jmath} \bar{j}}^{\rho}$ give the contributions $\mathbf{M}_{j} \cdot \mathbf{M}_{j}$ and the 2-band couplings $c_{j \bar{\jmath}}^{\rho, \sigma}$ and $u_{j \bar{\jmath} j}^{\rho, \sigma}$ lead to the products $\mathbf{M}_{j} \cdot \mathbf{M}_{\bar{\jmath}}$. The 4-band couplings result in the other products $\mathbf{M}_{i} \cdot \mathbf{M}_{j}$. For $N$ large, all couplings take the same value $g_{i j}=g>0$ ( $s$-wave AFM).

As a result, all band pairs $(j, \bar{j})$ are interacting with each other. In particular, for $N$ odd, the band $r=$ $(N+1) / 2$ is interacting with all other band pairs and there is no qualitative difference between odd and even $N$. This contrasts the ladder-case at energies below $E_{c}$, where only interactions within the band pairs $(j, \bar{\jmath})$ are present, respectively for odd $N$, within the band $r$. This then leads to an odd-even effect, i.e., the band $r$ present only for odd $N$ exhibits a gapless spinon-mode [19].

It is instructive to Fourier transform Eq. (36) 


$$
\begin{aligned}
H= & H_{0}-\frac{1}{2} \sum_{i, j} g_{i j} \\
& \times \sum_{k, k^{\prime}, q}\left[\Psi_{R i s_{1}}^{\dagger}(k) \tau_{s_{1} s_{1}^{\prime}}^{p} \Psi_{L \bar{\imath} s_{1}^{\prime}}(k+q)+\text { H.c. }\right] \\
& \times\left[\Psi_{R j s_{2}}^{\dagger}\left(k^{\prime}\right) \tau_{s_{2} s_{2}^{\prime}}^{p} \Psi_{L \bar{\jmath} s_{2}^{\prime}}\left(k^{\prime}-q\right)+\text { H.c. }\right]
\end{aligned}
$$

The Fourier transformed 2D AFM Heisenberg Hamiltonian (large- $U$ limit) takes the form

$$
\begin{aligned}
H_{J}= & -J \sum_{\mathbf{k}, \mathbf{k}^{\prime}, \mathbf{q}}\left(e^{i q_{x}}+e^{i q_{y}}\right) \\
& \times \Psi_{s_{1}}^{\dagger}(\mathbf{k}) \tau_{s_{1} s_{1}^{\prime}}^{p} \Psi_{s_{1}^{\prime}}(\mathbf{k}+(\pi, \pi)+\mathbf{q}) \\
& \times \Psi_{s_{2}}^{\dagger}\left(\mathbf{k}^{\prime}\right) \tau_{s_{2} s_{2}^{\prime}}^{p} \Psi_{s_{2}^{\prime}}\left(\mathbf{k}^{\prime}-(\pi, \pi)-\mathbf{q}\right)
\end{aligned}
$$

where we substituted $\mathbf{q}$ by $\mathbf{q}+(\pi, \pi)$. Therefore, the interacting part of the Hubbard Hamiltonian (37) is basically the Heisenberg Hamiltonian restricted to the umklapp surface (the operators $\Psi_{R j s}^{\dagger}$ and $\Psi_{L \bar{\jmath} s}$ have a momentum difference of $\pi$ ). Since $U / t$ is small (and therefore the energies low), there is no $q$-dependent coupling in Eq. (37). In real space, this corresponds to long-range spin-spin interactions. Note that the generalization/crossover of the Hamiltonian (37) to $2 \mathrm{D}$ is straightforward. Introducing a sum over $q_{y}$ is sufficient $\left(q=q_{x}\right)$.

To conclude this section: The half-filled $N$-leg Hubbard Hamiltonian — with on-site repulsion between electrons - becomes at energies below the gap $\Delta$ a Hamiltonian with purely long-range AFM spin-spin interactions.

\section{Physical properties: Bosonization}

Using bosonization techniques, we derive the physical properties of the AFM Hamiltonian, Eq. (36).

\section{Bosonized Hamiltonian}

The noninteracting part is given by $\tilde{H}_{0}$, see Eq. (15). At the AFM fixed-point, the interacting part is as follows. The 4-band interactions take the form,

$$
\mathcal{H}_{j k}^{4 B}=-g_{j k} \mathcal{H}_{C}\left(2 \mathcal{H}_{S 1}+\mathcal{H}_{S 2}\right)+(j \leftrightarrow \bar{\jmath}, k \leftrightarrow \bar{k}) .
$$

The $\mathcal{H}_{S 1}$ contains the spin-fields $\Phi_{\sigma j \bar{\jmath}-}$ and $\theta_{\sigma j \bar{\jmath}+}$,

$$
\mathcal{H}_{S 1}=\cos \left[\beta\left(\Phi_{\sigma j \bar{\jmath}-}-\Phi_{\sigma k \bar{k}-}+\theta_{\sigma k \bar{k}+}-\theta_{\sigma j \bar{\jmath}+}\right)\right],
$$

where $\beta=\sqrt{\pi}$ and $\mathcal{H}_{S 2}$ contains the spin-fields $\Phi_{\sigma j \bar{\jmath}+}$ and $\theta_{\sigma j \bar{\jmath}-}$,

$$
\begin{aligned}
\mathcal{H}_{S 2} & =\cos \left[\beta\left(\theta_{\sigma j \bar{\jmath}-}-\theta_{\sigma k \bar{k}-}+\Phi_{\sigma k \bar{k}+}-\Phi_{\sigma j \bar{\jmath}+}\right)\right] \\
& +\cos \left[\beta\left(\theta_{\sigma j \bar{\jmath}-}+\theta_{\sigma k \bar{k}-}-\Phi_{\sigma k \bar{k}+}-\Phi_{\sigma j \bar{\jmath}+}\right)\right] .
\end{aligned}
$$

The charge-part, $\mathcal{H}_{C}$, includes the charge-fields $\Phi_{\rho j \bar{\jmath}+}$ and $\theta_{\rho j \bar{\jmath}-}$,

$$
\begin{aligned}
\mathcal{H}_{C}= & \cos \left[\beta\left(\Phi_{\rho k \bar{k}+}-\Phi_{\rho j \bar{\jmath}+}+\theta_{\rho j \bar{\jmath}-}-\theta_{\rho k \bar{k}-}\right)\right] \\
& +\cos \left[\beta\left(\Phi_{\rho k \bar{k}+}+\Phi_{\rho j \bar{\jmath}+}-\theta_{\rho j \bar{\jmath}-}-\theta_{\rho k \bar{k}-}\right)\right] .
\end{aligned}
$$

In $\mathcal{H}_{C}$, the first cosine comes from the 4-band nonumklapp interactions (33), while the second cosine results from the 4-band umklapp interactions (34). The bosonized form of the 2-band non-umklapp terms is

$$
\begin{aligned}
\mathcal{H}_{j, 1}^{2 B}= & g_{j} \cos \left(2 \beta \theta_{\rho j \bar{\jmath}-}\right)\left[2 \cos \left(2 \beta \theta_{\sigma j \bar{\jmath}-}\right)\right. \\
& \left.-2 \cos \left(2 \beta \Phi_{\sigma j \bar{\jmath}+}\right)-4 \cos \left(2 \beta \Phi_{\sigma j \bar{\jmath}-}\right)\right] \\
& +2 g_{j} \cos \left(2 \beta \Phi_{\sigma j \bar{\jmath}+}\right) \cos \left(2 \beta \theta_{\sigma j \bar{\jmath}-}\right)
\end{aligned}
$$

and of the 2-band umklapp terms

$$
\begin{aligned}
\mathcal{H}_{j, 2}^{2 B}= & g_{j} \cos \left(2 \beta \Phi_{\rho j \bar{\jmath}+}\right)\left[2 \cos \left(2 \beta \theta_{\sigma j \bar{\jmath}-}\right)\right. \\
& \left.-2 \cos \left(2 \beta \Phi_{\sigma j \bar{\jmath}+}\right)-4 \cos \left(2 \beta \Phi_{\sigma j \bar{\jmath}-}\right)\right] \\
& -3 g_{j} \cos \left(2 \beta \Phi_{\rho j \bar{\jmath}+}\right) \cos \left(2 \beta \theta_{\rho j \bar{\jmath}-}\right) .
\end{aligned}
$$

The LLPs at the AFM fixed-point take the form

$$
K_{\rho j \pm}=\sqrt{\frac{\pi v_{j} \mp 3 g_{j} / 4}{\pi v_{j} \pm 3 g_{j} / 4}}
$$

(implying $K_{\rho j+}<1$ and $K_{\rho j-}>1$ ) and

$$
K_{\sigma j \pm}=\sqrt{\frac{\pi v_{j} \pm g_{j} / 4}{\pi v_{j} \mp g_{j} / 4}},
$$

i.e., $K_{\sigma j+}>1$ and $K_{\sigma j-}<1$. Table II gives an overview about the LLPs in the AFM and ISL phase.

Note that the commutation relation for the field and its dual field hinders the pinning, i.e., the localization of the field $\Phi_{\alpha}$ and its dual field $\theta_{\alpha}$ in the minimum of a cosine at the same time.

TABLE II. The phase at a particular energy-scale is related to the value of the LLPs at this scale. The table shows the LLPs in the insulating AFM, ISL, and superconducting (SC) phase. Note that always $K_{\rho j-}>1$ and $K_{\sigma j-}<1$. The differences are, that $K_{\rho j+}<1$ for the AFM and ISL and $K_{\sigma j+}<1$ for the ISL and the SC.

\begin{tabular}{lcccc}
\hline \hline & $K_{\rho j+}$ & $K_{\rho j-}$ & $K_{\sigma j+}$ & $K_{\sigma j-}$ \\
\hline AFM & $<1$ & $>1$ & $>1$ & $<1$ \\
ISL & $<1$ & $>1$ & $<1$ & $<1$ \\
SC & $>1$ & $>1$ & $<1$ & $<1$ \\
\hline \hline
\end{tabular}




\section{Charge-sector}

For the charge-sector, we then find, that the same fields are pinned as in the half-filled two-leg (respectively $N$-leg) Hubbard ladder [19,26], i.e., $\Phi_{\rho j \bar{\jmath}+} \approx 0$ and $\theta_{\rho j \bar{\jmath}-} \approx 0$. The type of Mott insulator is thus the same in $1 \mathrm{D}$ and $2 \mathrm{D}$. Note that pinning of $\Phi_{\rho j \bar{\jmath}-}$ instead of $\theta_{\rho j \bar{\jmath}-}$ leads to another type of insulator. The difference between these two types of insulators becomes physically relevant upon (hole) doping. While the second type most likely becomes a FL, the first type becomes (in case we have a spin-gap) a superconductor, since doping only depins the $\Phi_{\rho}$-fields but does not destroy the phase coherence, i.e., the $\theta_{\rho}$-fields remain pinned, see Ref. [19].

\section{Spin-sector}

In contrast, in the spin-sector, both the fields $\Phi_{\sigma j \bar{j}}$ and the dual fields $\theta_{\sigma j \bar{\jmath} \pm}$ appear in a cosine, resulting in a competition between different "phases", i.e., pinning of the field vs. its dual field. However, since $K_{\sigma j+}>1$ and $K_{\sigma j-}<1$ (for a comparison, see Sec. III A), it is more favorable to pin $\theta_{\sigma j \bar{\jmath}+}$ and $\Phi_{\sigma j \bar{\jmath}-}$ than the corresponding dual fields.

The physical interpretation is then the following. The pinning of $\Phi_{\sigma j \bar{\jmath}-}$ leads to a spinon confinement, leaving as physical particles spin 1 magnons. Note that only the differences $\theta_{\sigma j \bar{\jmath}+}-\theta_{\sigma k \bar{k}+}$ appear in $H^{4 B}$, such that the total magnon mode(s), given by $\theta_{T}=\sqrt{2 / N} \sum_{j} \theta_{\sigma j \bar{\jmath}+}$ and $\Phi_{T}=\sqrt{2 / N} \sum_{j} \Phi_{\sigma j \bar{\jmath}+}$, remains gapless. The $\pi$ and 0 mode then result from a superposition of left/right going modes along the chains (since we use open boundary conditions, the transvers momentum is always positive).

For the calculation of the spin-spin correlation function, we first rewrite the real-space operators $d_{i s}$ in terms of the band operators $\Psi_{h j s}$ and then the band operators in terms of the bose-operators. Using that only products of fields, which contain the pinned charge-fields $\Phi_{\rho j \bar{\jmath}+}$ and $\theta_{\rho j \bar{\jmath}}$ - give non-vanishing contributions to the spinspin correlation function, the real-space spin-operator at the position $(x, i)$ takes the form

$$
\begin{array}{r}
S_{i}^{p}(x)=\frac{1}{2} \sum_{j} \gamma_{i j} \gamma_{i \bar{\jmath}}\left(\Psi_{R j s}^{\dagger} \tau_{s s^{\prime}}^{p} \Psi_{L \bar{\jmath} s^{\prime}} e^{-i\left(k_{F j}+k_{F \bar{\jmath}}\right) x}\right. \\
+R \leftrightarrow L) \\
+R)
\end{array}
$$

where $\gamma_{j m}=\sqrt{2 /(N+1)} \sin [\pi j m /(N+1)]$ and at half-filling, $k_{F j}+k_{F \bar{\jmath}}=\pi$. The abelian bosonization scheme used here, breaks non-abelian symmetries, i.e., the $\mathrm{SU}(2)$ spin-symmetry is broken down to $\mathrm{U}(1)$, see, e.g., Refs. 22, 29. In our case, only the $x$ and $y$ components of the spin-spin correlation function give then straightforwardly the correct physical result (this "problem" occurs also in a single chain with a spin-gap [22]).
The products of fields appearing in Eq. (47) are then rewritten in terms of bose-operators according to

$$
\Psi_{R j \uparrow}^{\dagger} \Psi_{L \bar{\jmath} \downarrow} \propto e^{i \sqrt{\pi}\left(-\Phi_{\rho j \bar{\jmath}+}-\Phi_{\sigma j \bar{\jmath}-}+\theta_{\rho j \bar{\jmath}-}+\theta_{\sigma j \bar{\jmath}+}\right)} .
$$

The charge-fields and $\Phi_{\sigma j \bar{\jmath}-}$ are pinned and can be set to zero. Using that $\theta_{\sigma j \bar{\jmath}+}-\theta_{\sigma k \bar{k}+} \approx 0$, we express $\theta_{\sigma j \bar{\jmath}+}$ in terms of the total spin-mode $\theta_{T}, \theta_{\sigma j \bar{\jmath}+}=\sqrt{2 / N} \theta_{T}$. Since $H_{0}$ is gaussian in the fields $\Phi_{T}$ and $\theta_{T}$, the spin-spin correlation function takes the form (for details about such calculations, consult, e.g., Ref. 27)

$$
\left\langle\mathbf{S}_{i}(x) \cdot \mathbf{S}_{l}(0)\right\rangle \propto(-1)^{i+l} \cos (\pi x) / x^{1 / N},
$$

where $x<\xi_{c} \propto 1 / E_{c}$ (for $x>\xi_{c}$ we are in the ISL phase, where the decay is exponentially with coherence length $\xi_{A F} \sim \xi_{c}$, respectively, for $N$ odd, $\left.\propto 1 / x\right)$.

The energy-scales for charge and spin-excitations are different. The LLPs in the charge-sector, see Eq. (45), deviate more from their noninteracting value $(=1)$ than the LLPs in the spin-sector, Eq. (46), i.e.,

$\left|K_{\rho j \pm}-1\right| \approx 3 g_{j} /\left(4 \pi v_{j}\right),\left|K_{\sigma j \pm}-1\right| \approx g_{j} /\left(4 \pi v_{j}\right)$

This implies that the charge-fields are pinned more strongly than the spin-fields and therefore the Mott-gap (we may interpret the RG-scale $\Delta$ as the charge-gap) is larger than the gap to spin-1/2 (spinon) excitations. The coupling between the different bands makes it difficult to investigate the detailed excitation spectrum, such that we do not go beyond this qualitative result (for the pure sine-Gordon model, there exist rigorous results about the gap dependence on $K_{\alpha}$, see Ref. 27]).

\section{EFFECT OF (HOLE) DOPING}

Next, we study the effect of doping away from halffilling. There are two different doping regimes. The lightly doped case, where the influence of the umklapp processes has to be taken into account, can be treated as a perturbation of the half-filled low-energy Hamiltonian (limit $\delta \rightarrow 0$ and $U$ finite). For increasing doping, one has then again to investigate the RG flow.

For the lightly doped case (Sec. V A), we find above $E_{c}$ the same phase as below $E_{c}$ (see Ref. [19]): a conducting spin-liquid (bound hole-pairs), see also Fig. 11. Furthermore, while at half-filling the magnetic correlations are only at $(\pi, \pi)$, they become incommensurate upon doping.

In Sec. V B, we briefly show how and why for increasing doping phase-coherence between all band pairs sets in and the system becomes a 2D-like $d$-wave superconductor. The low-energy Hamiltonian takes the form of a $d$-wave BCS model, where the pairing is between electrons.

Note that the superconducting instability is for $N \gg 2$ generated in the same way as for the $2 \mathrm{D}$ case [14 16]: 
A Kohn-Luttinger-type attraction [23] is mediated by AFM processes [24. In contrast, the superconducting instability in the two-leg ladder is generated by forwardscattering processes [18].

\section{A. Lightly doped case}

The ISL phase of the half-filled two-leg Hubbard ladder becomes superconducting when doped, because of the phase-coherence (see discussion of the charge-sector in the previous section) and the spin-gap present at halffilling [26]. Here, we show that the doped Hubbard AFM becomes a conducting spin-liquid, formed by bound hole-pairs (this phase may becomes superconducting at very low energies; for the 4-leg ladder, see discussion in Ref. [19]).

The 2-band interactions alone, see Eqs. (43) and 44), would result in an ISL phase, i.e., $\Phi_{\sigma j \bar{j} \pm} \approx 0$. It is the presence of interactions between bands $i$ and $j$ which are not "paired" $(j \neq i, \bar{\imath})$, which renders the system a $2 \mathrm{D}$ like AFM. The effect of a decreasing $N<\infty$ is therefore that interactions between unpaired bands become suppressed and the AFM correlations are reduced. Next, we show that the effect of doping away from half-filling is a similar one, i.e., the doping $\delta$ corresponds to $1 / N$.

At low dopings and at finite $N$, we can dope the system perturbatively by introducing a chemical potential term $-\mu Q$ ( $Q$ is the total charge) in the half-filled low-energy Hamiltonian, see Ref. [19]. For the charge-sector, we can use bosonization, where the charge density is given by $\rho=\sum_{j} \partial_{x} \Phi_{\rho j}$
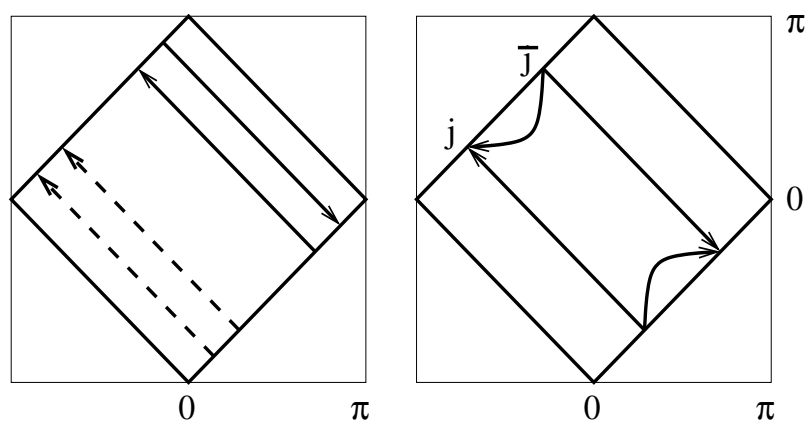

FIG. 4. The square is the umklapp surface, which is the FS at half-filling $\left(t=t_{\perp}\right)$. Left: There are 2 types of AFM processes; AFM processes which are umklapp processes (dashed arrows), and AFM processes which are not umklapp processes (solid arrows). Right: The (non-umklapp) AFM processes which take place within a band pair $(j, \bar{\jmath})$ are identical to the corresponding ( $d$-wave) Cooper processes within this band pair. Upon doping away from half-filling, these processes remain large, open a spin-gap and lead to phase coherence within band pairs $(j, \bar{\jmath})$. Note that we use for the ladders open boundary conditions, whereas the above figures are drawn - for a better comparison with the $2 \mathrm{D}$ case - for periodic boundary conditions.
Doping then introduces kinks in the fields $\Phi_{\rho j \bar{\jmath}+}$, such that the expectation value of cosine terms, which contain this field gradually goes to zero (the kink is a fermionic, strongly localized particle). Here, the kinks are bound hole-pairs of charge 2 and zero spin. All 4-band interactions contain the field $\Phi_{\rho j \bar{\jmath}}$, see Eq. (42), and therefore vanish. The effect of the doping on the 2-band interactions is the same as when doping an ISL, i.e., in the bosonized version, the umklapp term (44) vanishes leaving only the term (43), which opens a spin-gap, and leads to $d$-wave-like phase coherence between the bands $j$ and $\bar{\jmath}$, corresponding to the Fermi momenta $\left(k_{F j}, \pi-k_{F j}\right)$ and $\left(\pi-k_{F j}, k_{F j}\right)$. The charge-gaps close to $(\pi / 2, \pi / 2)$ are the smallest ones (note that $g_{N / 2} / v_{N / 2}<\ldots<g_{1} / v_{1}$ ), such that the hole-pairs enter first there.

In other words, it is the fact, that the ( $s$-wave) AFM processes

$$
-\mathbf{M}_{j} \cdot \mathbf{M}_{\bar{\jmath}}=-\Psi_{R j \downarrow}^{\dagger} \Psi_{L \bar{\jmath} \uparrow} \Psi_{L j \uparrow}^{\dagger} \Psi_{R \bar{\jmath} \downarrow} \text { (+other terms) }
$$

(partially) coincide with the ( $d$-wave) Cooper processes with momentum transfer $\pi$

$$
\Delta_{j}^{\dagger} \Delta_{\bar{\jmath}}=\Psi_{R j \downarrow}^{\dagger} \Psi_{L j \uparrow}^{\dagger} \Psi_{L \bar{\jmath} \uparrow} \Psi_{R \bar{\jmath} \downarrow} \text { (+other terms), }
$$

which makes a doped AFM a superconductor: These particular AFM/Cooper processes flow at higher energies - driven by AFM processes - to strong coupling and are not suppressed at lower energies by doping (see also Fig. (4). We therefore have AFM mediated superconductivity 24. The full phase-coherence around the FS then grows out of the phase-coherence between bands $j$ and $\bar{\jmath}$, see below.

Upon doping, the AFM correlations become incommensurate. When doping away from half-filling, $k_{F j}+$ $k_{F \bar{\jmath}}<\pi$, and the AFM peak shifts away from $(\pi, \pi)$, see Eq. (47). In particular, for small $t_{\perp} / t \ll 1$, all bands are (almost) equally doped and $k_{F j}+k_{F \bar{\jmath}}=\pi(1-\delta)$, i.e., the incommensurability is equal the doping $\delta$. Furthermore, doping leads to a cutoff of the AFM processes at the energy-scale of the chemical potential $\mu$ and therefore to an exponential decay of the spin-spin correlation function, where the coherence-length is given by $\xi_{A F} \propto 1 / \mu$.

\section{B. Increasing doping}

We argue, that for increasing doping, phase-coherence between the band pairs sets in, when the "distance" between neighboring bands is of the order of the "distance" of the FS to the umklapp surface, i.e., for dopings $\delta>\delta_{c}(N) \sim\left(t_{\perp} / t\right) / N$. The system then becomes a 2D-like $d$-wave superconductor.

Since a finite chemical potential $\mu$ results in a lowenergy cutoff for the umklapp and 4-band-AFM interactions, one has to start initially with all interactions which are large at this cutoff energy-scale. In leading order, this 
implies that not only the 4-band interactions $c_{j k \bar{k} \bar{j}}^{\rho, \sigma}$ and $u_{j k \bar{k} \bar{j}}^{\rho, \sigma}$ have to be taken into account, but also "neighbor" processes of the form $c_{j k \bar{k} \pm 1 \bar{\jmath} \pm 1}^{\rho, \sigma}$ and $u_{j k \bar{k} \pm 1 \bar{\jmath} \pm 1}^{\rho, \sigma}$. These processes are only present down to an energy-scale $t_{\perp} / N$. For dopings $0<\delta<\left(t_{\perp} / t\right) / N$, the "neighbor" processes are cutoff before the main AFM processes, which leads to a decoupling into band pairs $(j, \bar{\jmath})$, while for $\delta>\left(t_{\perp} / t\right) / N$ all AFM processes are cutoff by the chemical potential. In this case, not only 2-band Cooper interactions within band pairs $(j, \bar{\jmath})$ are large at the cutoff energy-scale, but also the neighboring 2-band Cooper interactions $(j, \bar{\jmath} \pm 1)$.

Integrating the RGEs without AFM and umklapp processes, but with initial-values for the Cooper and forward interactions given by the AFM phase, see Eq. (27), and similar initial-values for non-leading Cooper processes $c_{j \bar{\jmath} \pm p}^{\rho, \sigma}(p=1,2,3, \ldots)$, we obtain a $d_{x^{2}-y^{2}}$ superconducting instability at lower energies. We always find that the scaling of the couplings is exactly such that (in particu$\left.\operatorname{lar}, 4 c_{i j}^{\rho}=c_{i j}^{\sigma}, f_{i j}^{\rho} \approx f_{i j}^{\sigma} \approx 0\right)$

$$
H=H_{0}+\sum_{i, j} \int d x V_{i j} \Delta_{i}^{\dagger} \Delta_{j},
$$

where the pairing operator is $\Delta_{j}=\Psi_{R j \uparrow} \Psi_{L j \downarrow}+\Psi_{L j \uparrow} \Psi_{R j \downarrow}$ and $V_{i j}<0$ for $i, j \leq N / 2$ respectively $i, j>N / 2$ and $V_{i j}>0$ in all other cases $\left(d_{x^{2}-y^{2}}\right.$ symmetry). The size (but not the sign) of $V_{i j}$ depends on the initial-values (i.e., on the doping). Note that the energy-scale of the system is reduced by doping, i.e., the superconductor has a lower scale than the AFM.

For large dopings, the 4-band interactions are cutoff before they could have flown sufficiently close to the AFM fixed-point, such that there is no instability in the RG flow and the low-energy phase is a FL 18.

As a result, for $N$ finite, there is between the insulating AFM respectively ISL phase at half-filling and the $d$-wave superconductor at higher doping, an intermediate, conducting phase with a spin-gap (see also Fig. 1). In the 2D-limit, the intermediate phase seems to disappear.

\section{CONCLUSIONS}

We have studied the dimensional crossover from $1 \mathrm{D}$ to $2 \mathrm{D}$ in half-filled, weakly interacting, $N$-leg Hubbard ladders for $t_{\perp}<t$. Using the RG method, we found a crossover energy $E_{c} \sim t \exp [-\alpha \exp (\gamma N)]$, where at energies below $E_{c}$ the behavior of the spin-sector is ladder-like and at energies above $E_{c} 2 \mathrm{D}$-AFM-like. We showed that the 4-band-AFM processes are responsible for an instability in the RG flow. Since without 4-band and umklapp processes the Hubbard ladders become a FL for large $N$ [18], AFM processes (at higher energies) are necessary and sufficient for a nontrivial phase in the (doped) $N$-leg Hubbard model.

In the AFM phase, the couplings flow towards universal ratios and we obtained an analytical expression for the Hamiltonian which is similar to the Heisenberg AFM. Using bosonization techniques, we further investigated this Hamiltonian and found that the charge-sector is the same as in the half-filled two-leg ladder, i.e., the type of Mott insulator is the same in 1D and 2D. For the spin-sector, bosonization techniques confirmed that the small- $U$ case has similar physical properties as the large- $U$ (Heisenberg) case.

The effects of doping on the half-filled Hubbard Hamiltonian and of reducing the number of coupled chains $N$ is (almost) the same: In both cases, the interactions between unpaired bands are suppressed, the antiferromagnetic correlations are reduced and only interactions within band pairs $(j, \bar{\jmath})$ remain. Doping then opens a spin-gap, but phase coherence exists only between bands $j$ and $\bar{\jmath}$. At higher doping levels $\delta \sim\left(t_{\perp} / t\right) / N$, we can expect that phase coherence between all bands sets in, such that the system becomes a 2D-like $d$-wave superconductor. While the lightly doped case is conveniently described by bound hole-pairs, the 2D-like superconductor at higher dopings is represented by a BCS-like $d$-wave Hamiltonian with electron pairing. In the doped case the antiferromagnetic correlations become incommensurate.

As a result, the Hubbard model cannot be understood in terms of an AFM, ISL or superconductor alone. The phase (correlation function) depends always on the energy-scale (respectively length-scale) and is related to the value of the LLPs. The AFM exhibits the highest energy-scale. At half-filling, the groundstate (for $N$ finite) is the ISL and for sufficient doping the $d$-wave superconductor. We conjecture that in the doped case, the flow of the couplings from the AFM to the $d$-wave takes place via an intermediate phase: Consult table II; AFM and superconductor differ in two LLPs. This intermediate (finite energy) phase has then the same LLPs as the ISL - suggesting that the spin-gap opens before phase coherence sets in.

We believe, that also for other systems of interacting electrons the dimensional crossover from $1 \mathrm{D}$ to $2 \mathrm{D}$ can be studied in a similar way and maybe enlightens our understanding of the 2D case.

\section{ACKNOWLEDGMENTS}

I am grateful to B. Binz, C. Honerkamp, K. Le Hur, T.M. Rice, and M. Sigrist for fruitful discussions and comments throughout this work.

[1] E. Dagotto and T.M. Rice, Science 271, 618 (1996).

[2] S. Chakravarty, B.I. Halperin, and D.R. Nelson, Phys. Rev. B 39, 2344 (1989). 
[3] For a review, see E. Manousakis, Rev. Mod. Phys. 63, 1 (1991).

[4] J.M. Luttinger, J. Math. Phys. 4, 1154 (1963).

[5] F.D.M. Haldane, J. Phys. C 14, 2585 (1981).

[6] S. Chakravarty, Phys. Rev. Lett. 77, 4446 (1996).

[7] For an introduction, see H.J. Schulz, in Correlated Fermions and Transport in Mesoscopic Systems, edited by T. Martin et al. (Ed. Frontiers, Gif-sur-Yvette, 1996, p. 81), and references therein.

[8] C. Castellani, C. Di Castro, and W. Metzner, Phys. Rev. Lett. 72, 316 (1994).

[9] D. Boies, C. Bourbonnais, and A.M.S. Tremblay, Phys. Rev. Lett. 74, 968 (1995).

[10] E. Arrigoni, Phys. Rev. Lett. 83, 128 (1999).

[11] P.W. Anderson, Phys. Rev. Lett. 64, 1839 (1990).

[12] An exception are strongly anisotropic systems, see A. Vishwanath and D. Carpentier, Phys. Rev. Lett. 86, 676 (2001).

[13] R. Shankar, Rev. Mod. Phys. 66, 129 (1994).

[14] D. Zanchi and H.J. Schulz, Phys. Rev. B 54, 9509 (1996); Europhys. Lett. 44, 235 (1998); Phys. Rev. B 61, 13609 (2000).

[15] C.J. Halboth and W. Metzner, Phys. Rev. B 61, 7364 (2000); Phys. Rev. Lett. 85, 5162 (2000).

[16] C. Honerkamp, M. Salmhofer, N. Furukawa, and T.M. Rice, Phys. Rev. B 63, 035109 (2001).
[17] B. Binz, D. Baeriswyl, and B. Douçot, condmat/0104424.

[18] H. Lin, L. Balents, and M. Fisher, Phys. Rev. B 56, 6569 (1997).

[19] U. Ledermann, K. Le Hur, and T.M. Rice, Phys. Rev. B 62, 16383 (2000).

[20] A. Auerbach, in Interacting Electrons and Quantum Magnetism (Springer, New York, 1994).

[21] H.J. Schulz, in Proceedings of Les Houches Summer School LXI, edited by E. Akkermans et al. (Elsevier, Amsterdam, 1995).

[22] J. Voit, Rep. Prog. Phys. 58, 977 (1995).

[23] W. Kohn and J.M. Luttinger, Phys. Rev. Lett. 15, 524 (1965).

[24] K. Miyake, S. Schmitt-Rink, and C.M. Varma, Phys. Rev. B 34, 6554 (1986); D.J. Scalapino, E. Loh, and J.E. Hirsch, Phys. Rev. B 34, 8190 (1986).

[25] For a related work with flat Fermi surface, see S. Dusuel, F. Vistulo de Abreu, and B. Douçot, cond-mat/0107548.

[26] H. Lin, L. Balents, and M. Fisher, Phys. Rev. B 58, 1794 (1998).

[27] A.O. Gogolin, A.A. Nersesyan, and A.M. Tsvelik, in Bosonization and Strongly Correlated Systems (Cambridge University Press, Cambridge, 1998).

[28] U. Ledermann, Ph.D. thesis, ETH Zürich (2001).

[29] E. Witten, Commun. Math. Phys. 92, 455 (1984). 